\title{
Face Mask Leak Determines Aerosol Delivery in Noninvasive Ventilation
}

\author{
Alexandra Haw, Michael McPeck, Ann D Cuccia, and Gerald C Smaldone
}

\begin{abstract}
BACKGROUND: Aerosol transport during noninvasive ventilation follows the flow of pressurized gas through the noninvasive ventilation circuit, vented via leak port and face mask, and inhaled by the patient. Recommendations for nebulizer placement are based on in vitro models that have focused primarily on aerosol losses via the leak port; face mask leaks have been avoided. This study tested aerosol delivery in the setting of controlled face mask leak. METHODS: Three nebulizer technologies were studied on a bench model using a lung simulator with a face mask placed onto a manikin head. Radiolabeled aerosol delivery (ie, inhaled mass) was determined by mass balance using filters and a gamma camera that tested the effects of nebulizer location and face mask leak. Low $(15-20 \mathrm{~L} / \mathrm{min})$ and high $(55-60 \mathrm{~L} / \mathrm{min})$ mask leaks were used to mimic realistic clinical conditions. RESULTS: Inhaled mass (\% nebulizer charge) was a function of nebulizer technology (with the nebulizer at ventilator outlet position: Aerogen $22.8 \%$, InspiRx $11.1 \%$, and Hudson $8.1 \% ; P=.001)$. The location of the nebulizer before or after the leak port was not important $(P=0.13$ at low leak and $P=0.38$ at high leak). Aerosol delivery was minimal with high mask leak (inhaled mass 1.5-7.0\%). Aerosol losses at the leak port at low mask leak were $28-36 \%$ versus $9-24 \%$ at high mask leak. Aerosol losses via the mask leak were 16-20\% at low mask leak versus 46-72\% at high mask leak. Furthermore, high face mask leak led to significant deposition on the mask and face (eg, up to $50 \%$ of the nebulizer charge with the Aerogen mask). CONCLUSIONS: During noninvasive ventilation, nebulizer placement at the ventilator outlet, which is a more practical position, is effective and minimizes deposition on face and mask. Aerosol therapy should be avoided when there is high face mask leak. Key words: aerosols; nebulizers and vaporizers; administration; inhalation; noninvasive ventilator; humidifiers; drug delivery. [Respir Care 2021;66(1):95-103. (C) 2021 Daedalus Enterprises]
\end{abstract}

\section{Introduction}

Noninvasive ventilation (NIV) is commonly used to support patients who develop acute respiratory failure nearing intubation. Aerosol treatments, delivered via inline nebulizers, are often used to improve lung function and treat exacerbations. To assist ventilation, NIV provides a pressurized stream of gas to the patient, and aerosols, by necessity, are

Dr Haw, Dr Smaldone, and Mr McPeck are affiliated with the Division of Pulmonary, Critical Care, and Sleep Medicine, Department of Medicine, Stony Brook University Medical Center, Stony Brook, New York. Ms Cuccia is affiliated with the Respiratory Care Program, School of Health Technology and Management, Stony Brook University, Stony Brook, New York.

Supplementary material related to this paper is available at http://www. rcjournal.com. transported by this gas and must follow complex paths to reach the patient's respiratory tract. To define drug delivery, investigators have devised models of NIV that facilitate the study of different parameters thought to affect aerosol behavior. Conventional noninvasive ventilators have a single limb circuit with an uncontrolled leak port, which is an obvious source of aerosol losses and has been the focus of many studies. ${ }^{1-6}$ Results of these studies have

\footnotetext{
The study was supported in part by InspiRx. Dr Smaldone and Ms Cuccia have disclosed relationships with InspiRx. Dr Haw and Mr McPeck have disclosed no conflicts of interest.

Correspondence: Alexandra Haw MD, Division of Pulmonary, Critical Care, and Sleep Medicine, 101 Nicolls Road, T17-040, Health Sciences Center, Stony Brook, NY 11794. E-mail: alexandra.haw@stonybrookmedicine.edu.
}

DOI: $10.4187 /$ respcare.07915 


\section{Face Mask Leak and Aerosol Delivery in NIV}

been variable with regard to the optimal nebulizer location, but a general consensus in the literature has emerged suggesting placement of the nebulizer between the mask and leak port (ie, in a pre-mask position, as seen in Fig. 1). The practicality of this nebulizer position is open to question. First, devices may need external support for optimal positioning and function. In addition, patient movement and comfort may be important factors requiring direct supervision. Modeling these factors in vitro is difficult. To our knowledge, mask leak, as opposed to the intentional leak at the leak port, has not been studied. Investigators have chosen to control the mask leak variable by eliminating it, usually by sealing the mask to a manikin or endplate. ${ }^{7,8}$ In this study, we created a bench model to measure the effectiveness of the nebulizer position in the presence of a defined face mask leak. The protocol characterized aerosol delivery in an adult NIV model with the nebulizer positioned either at the ventilator outlet or at the pre-mask position, and at a predefined mask leak described as low or high.

\section{Methods}

\section{Experimental Setup}

Figure 1 depicts the experimental setup that we used at in the Aerosol Laboratory at Stony Brook University Medical Center, Stony Brook, New York. Studies were performed using the Active Servo Lung (ASL 5000; IngMar Medical, Pittsburgh, Pennsylvania) breathing simulator with the V60 noninvasive ventilator (Philips Respironics, Murrysville, Pennsylvania). The V60, a single-limb, constant-flow, dedicated noninvasive ventilator was set to an inspiratory pressure of $15 \mathrm{~cm} \mathrm{H}_{2} \mathrm{O}$, an expiratory pressure of $5 \mathrm{~cm} \mathrm{H}_{2} \mathrm{O}$, an inspiratory time of $1.0 \mathrm{~s}$ in the spontaneous/timed (S/T) mode, and a backup rate of 5 breaths/min. To simulate a spontaneously breathing patient with COPD, the ASL 5000 was set at a breathing frequency of 18 breaths/min, a compliance of $60 \mathrm{~mL} / \mathrm{cm} \mathrm{H}_{2} \mathrm{O}$, an inspiratory resistance of $10 \mathrm{~cm} \mathrm{H}_{2} \mathrm{O} / \mathrm{L} / \mathrm{s}$, and an expiratory resistance of $15 \mathrm{~cm} \mathrm{H}_{2} \mathrm{O} / \mathrm{L} / \mathrm{s}$. The profile of the negative inspiratory pressure generated by the ASL 5000 at the start of a breath was created by setting $5 \%$ of respiratory cycle time to active inspiration, $3 \%$ to end-inspiratory hold, and $15 \%$ to return of pressure to baseline. ${ }^{3}$ A noninvasive circuit without humidification and with a "Leak 1" exhalation port (582073, Philips Respironics), was attached to a mediumsized NIV oronasal mask (AF531, Philips Respironics) that was fitted to a rigid plastic adult mannequin head (Fisher and Paykel Healthcare, Auckland, New Zealand) with a 22-mm inner diameter airway opening at the mouth position. Tapered probes were inserted underneath the mask cushion, between the mask and mannequin face (Fig. 1), to provide controlled mask "leak" of either 15-20 L/min (low leak) or

\section{QUICK LOOK}

\section{Current knowledge}

Aerosol delivery during noninvasive ventilation (NIV) has been investigated using bench models without incorporation of a face mask leak, which occurs frequently in the clinical setting. Previous studies have supported the consensus to place the nebulizer between the leak port and face mask (ie, a pre-mask position). This position is inconvenient clinically, both for patients and for health care providers. However, the presence of a mask leak may change the dynamics of aerosol flow through the NIV circuit and significantly influence aerosol delivery.

\section{What this paper contributes to our knowledge}

This is the first bench study to incorporate a deliberate low and high mask leak to assess the effects on aerosol dynamics and delivery in NIV. Aerosol delivery with the nebulizer positioned at the ventilator outlet was as effective as the pre-mask position. Placement of nebulizer at the ventilator outlet allows for more reliable aerosol delivery and convenience in a clinical setting, and it would minimize aerosol deposition on the face. Furthermore, high mask leak significantly decreased aerosol delivery and should be avoided during aerosol therapy.

50-60 L/min (high leak) as indicated on the V60 monitor. The same ASL 5000 and ventilator settings were used for all experiments.

We tested the following nebulizers: Aerogen Solo (Aerogen, Galway Ireland), InspiRx i-AIRE (InspiRx, Somerset, New Jersey), and Hudson MicroMist (Teleflex Medical, Morrisville, North Carolina). To initiate a test run, the ASL 5000 was turned on and breathed spontaneously, then the V60 was turned on and connected to the ASL 5000 and confirmed to be functioning properly. Using the tapered probes, the leak was adjusted, and then the nebulizer was turned on. The degree of mask leak was set before nebulization; leak measurements were observed to decrease with nebulizer flow. No changes in any settings were made after the start of nebulization. ASL 5000 volumes were unchanged with nebulization, and the V60 volume display readings were increased with nebulizer flow by $5-10 \%$ (i-AIRE and Hudson respectively).

\section{Study Protocol}

To study the path of aerosol delivery, in separate experiments, aerosol collection filters (Pari, Starnberg, Germany) were placed either in the pre-leak position (proximal to the 


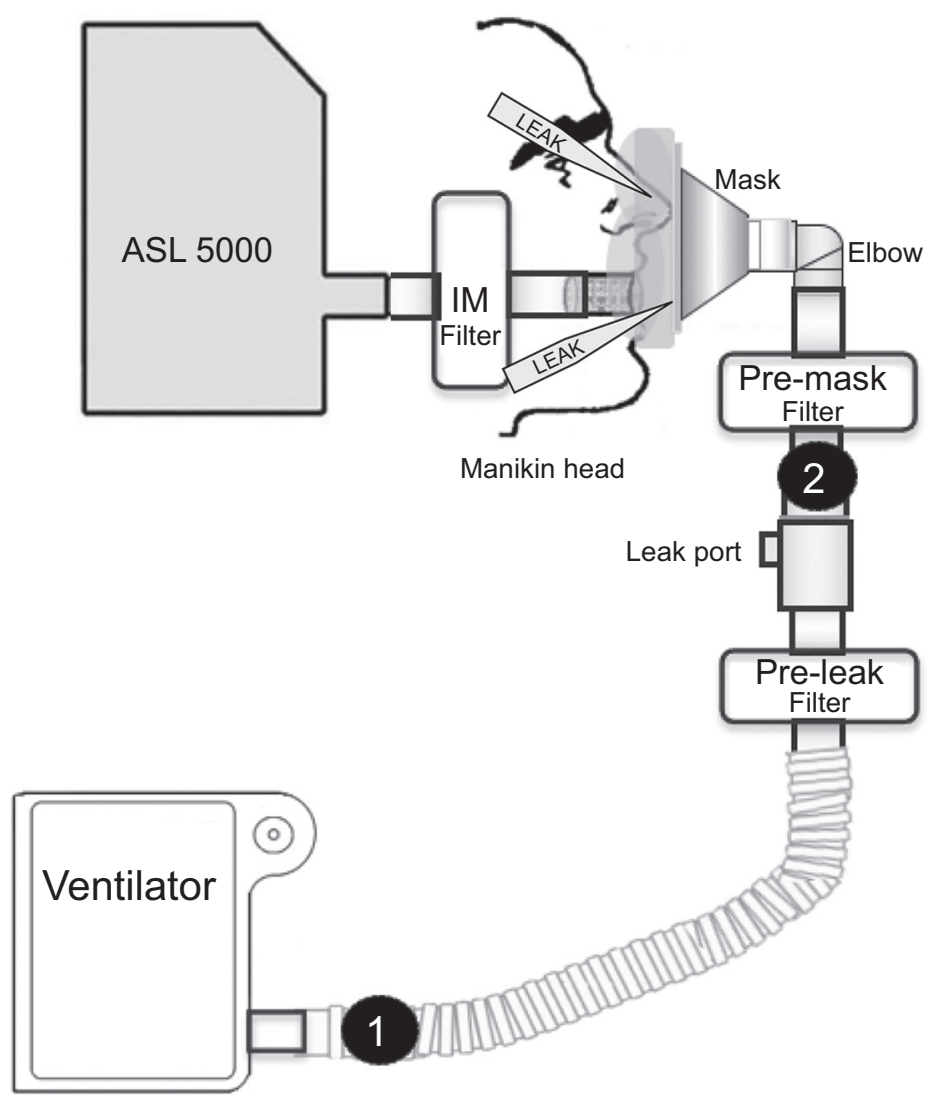

Fig. 1. Schematic diagram of the component parts of the experimental setup are shown starting with the Respironics V60 noninvasive ventilator at the bottom of the figure. The Ingmar ASL 5000 Active Servo Lung simulated a COPD breathing pattern. The direction of gas flow is from the V60 toward the ASL 5000. The oronasal mask was strapped onto the rigid plastic adult mannequin head and adjusted for mask leak using tapered probes and measured with the leak monitor on the V60. The 3 locations of aerosol-collection filters are labeled with their respective circuit positions: pre-leak filter (proximal to the leak port), pre-mask filter (proximal to the oronasal mask), and inhaled mass (IM) filter (inside the head of the manikin distal to the airway opening). Only one filter was in use at any given time, depending upon the test that was being conducted. The numbers denote the 2 nebulizer mounting positions used in the study: $1=$ ventilator outlet; $2=$ proximal to the pre-mask filter. Nebulizers mounted at these positions were maintained in their optimal operating orientations. A fourth filter (not shown), supplied with the noninvasive ventilator circuit, was placed at the ventilator outlet prior to attaching the circuit, per manufacturer instructions. The experimental setup was operated in a laboratory fume exhaust hood to scavenge radioactive aerosol escaping from the circuit leak port and the deliberate leaks created in the mask seal.

leak port), at the pre-mask position (between the mask and leak port), or inside the head (inhaled mass filter) distal to the airway opening, as shown in Figure 1. For each filter location, the nebulizer was placed at either the ventilator outlet or in the pre-mask position and tested at low mask leak and high mask leak. For each experimental configuration, 3 nebulizer types were tested, using multiple examples of each to test inter- and intra-device variability.

The Aerogen Solo, a vibrating mesh nebulizer (VMN), was operated with an Aerogen Pro-X Controller in its 30min treatment mode. The InspiRx i-AIRE breath-enhanced jet nebulizer (BEJN), powered by gas flow at $3.5 \mathrm{~L} / \mathrm{min}$, enhances aerosol generation using energy from the ventilator gas stream as it transits through the nebulizer. The Hudson MicroMist is a conventional sidestream, small-volume jet nebulizer $(\mathrm{JN})$ that requires a nebulizer flow of 8 $\mathrm{L} / \mathrm{min}$. Both the BEJN and the JN may be operated with air, oxygen, or blended gas, but we utilized dry medical air administered from a cylinder at $50 \mathrm{psig}$ with a back pressure compensated flow meter. Five Aerogen Solo nebulizers, 3 InspiRx i-AIRE nebulizers, and 3 Hudson MicroMist nebulizers were tested. More Aerogen units were tested than the other brands because of device failures.

Each nebulizer was charged with $3 \mathrm{~mL}$ of radiolabeled saline (technetium pertechnetate). All nebulizers were rotated throughout experiments to test reproducibility. The VMN nebulizer was positioned in the circuit using the Aerogen Adult T-piece, oriented according to manufacturer instructions, ${ }^{9}$ and operated with the Aerogen Pro-X Controller. The BEJN was attached directly to the ventilator outlet port with a compatible connector. The JN was attached to the NIV circuit with a Hudson spring-loaded T-adapter. While both the VMN and JN were tested in the pre-mask and ventilator outlet position, the BEJN was tested only in 

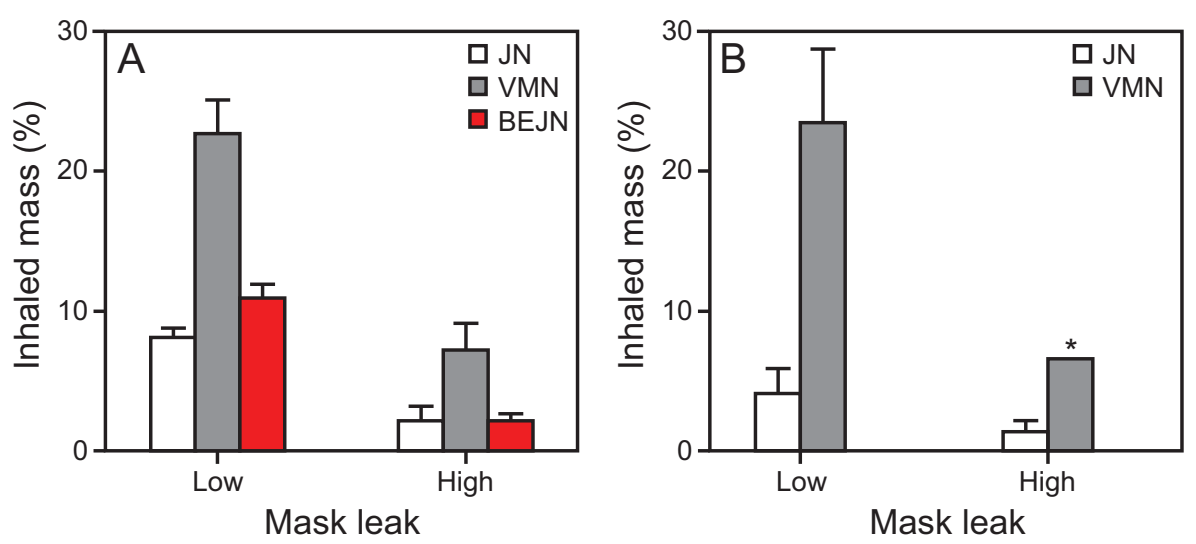

Fig. 2. Inhaled mass experiments with a nebulizer positioned $(A)$ at the ventilator outlet or $(B)$ in the pre-mask position. Inhaled mass was measured as a percentage of nebulizer charge. The bars represent average values, and the error bars reflect the standard deviation. There are significant inter-nebulizer differences $(P=.001)$ with VMN $>$ BEJN $>$ JN. Aerosol delivery was significantly reduced at high mask leak at both nebulizer positions $(P<.001)$. Aerosol delivery with the nebulizer placed $(A)$ at the ventilator outlet was equally or more effective than aerosol delivery to the inhaled mass filter when nebulizer was placed $(B)$ in the pre-mask position (low leak, $P=.13$; high leak, $P=.38$ ). ${ }^{*}$ Only 1 experiment for the VMN in the pre-mask position at high mask leak is reported because up to 5 experiments, with different VMN units, were performed in this configuration without success (nebulizer residual $>10 \%$ ). VMN = vibrating mesh nebulizer; $\mathrm{JN}=$ jet nebulizer; BEJN = breath-enhanced jet nebulizer.

the ventilator outlet position due to its design configuration and incompatibility with the pre-mask position.

A total of 95 experiments were performed to provide duplicate studies for each experimental condition. For all experiments, aerosol deposition onto the face and mask (combined) was measured. In addition, based on previous work from this laboratory, ${ }^{10,11}$ the interaction between the face mask and facial deposition was measured. Facial deposition was analyzed in a single subset of experiments using the Aerogen to demonstrate both the distribution and quantity of aerosol deposition onto the face and mask separately.

\section{Inhaled Mass and Mass Balance}

Aerosol delivery to the inhaled mass filter inside the head, plus each intermediate filter location, and a complete mass balance (including nebulizer residual, nebulizer adapter, and ventilator circuit tubing) was quantified and expressed as a percentage of nebulizer charge (see the supplementary materials at http://www.rcjournal.com). For each filter position (ie, pre-leak, pre-mask, and inhaled mass), a single filter was placed in the circuit for a given experiment. Radioactivity was measured via a gamma camera (Maxi Camera 400, General Electric, Horsholm, Denmark; Power Computing, Model 604/150/D, Austin, Texas; Nuclear MAC 4.2.2, Scientific Imaging, Thousand Oaks, California).

\section{Statistical Analysis}

Data were reported as mean $\pm \mathrm{SD}$. The Kruskal-Wallis test was used to detect differences in efficiency between the 3 nebulizers in the pre-leak and inhaled mass filter experiments, respectively. A Wilcoxon test was performed to determine if there were significant differences between nebulizer position (at the pre-mask versus ventilator outlet), whether there was a significant loss of aerosol through the leak port and mask leak, and whether the mask leak had an effect. The amount of aerosol lost through the leak port was measured by determining the difference in average aerosol deposition between the pre-leak filter (before the leak port) and the pre-mask filter (after the leak port). These results were normalized by expressing losses as a percentage of average aerosol deposited on the pre-leak filter, which represents $100 \%$ of aerosol that is available at that point in the circuit. The pre-leak filter measurement accounted for aerosol deposited in the circuit and in the adaptors from the ventilator to the leak port as well as residual left in the nebulizer. Similarly, the aerosol lost through the face mask was calculated by determining the difference between the average pre-mask filter (before the face mask) and average amount deposited on the face and mask as well as the inhaled mass filter. These measurements were normalized to the amount of aerosol deposited onto the pre-mask filter, which, in this configuration, defined the quantity of aerosol available for delivery to all structures downstream to this location. We used GraphPad Prism 7.0e for Mac OS (GraphPad Software, San Diego, California) to analyze the data and create the graphical representations of the data.

\section{Results}

\section{Inhaled Mass Filter Studies}

Comparing the efficiency of the 3 different nebulizers (Fig. 2), the VMN was the most efficient, followed by the BEJN and the JN. With the nebulizers at the ventilator 

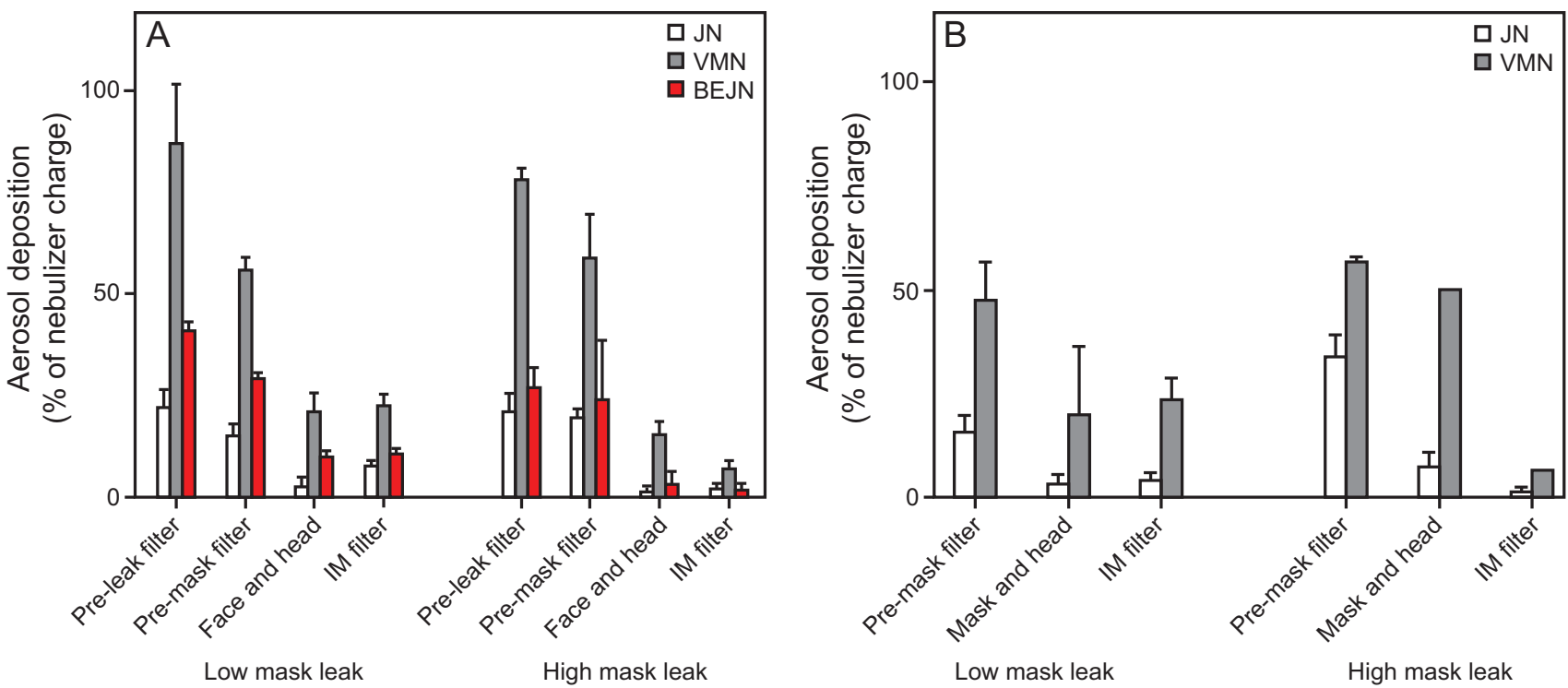

Fig. 3. Aerosol delivery as a percentage of nebulizer charge at different points on the noninvasive ventilator circuit. The bars represent average values, and the error bars reflect the standard deviation. Nebulizers were positioned at (A) the ventilator outlet or (B) the pre-mask position to deliver aerosol to the respective filters, with some aerosol depositing on the mask and face and the rest depositing in the inhaled mass filter. Between the pre-leak and pre-mask filter, aerosol was lost through the leak port. Between the pre-mask and inhaled mass filter, aerosol was lost by depositing on the mask and face or vented via the face mask leak. Notably, when the VMN was placed at the pre-mask position with a high mask leak, $\sim 50 \%$ of the nebulizer charge deposited on the mask and face. With the nebulizers at the ventilator outlet, there was higher circuit deposition with the VMN (5.0-20\%) compared to the JN (2.0-8.0\%) and BEJN (2.0-6.0\%). VMN = vibrating mesh nebulizer; JN = jet nebulizer; $\mathrm{BEJN}=$ breath-enhanced jet nebulizer.

outlet at low and high mask leak, inter-nebulizer differences were observed with $P$ values of .001 and .040 , respectively. At low mask leak, the VMN delivered (mean \pm SD) $22.8 \pm 2.3 \%$ of the nebulizer charge to the inhaled mass filter, whereas the BEJN delivered $11.1 \pm 0.9 \%$ and the JN delivered $8.1 \pm 0.2 \%$. Nebulizer residual defined device efficiency, with lower nebulizer residual indicating better nebulizer efficiency (VMN $5.6 \pm 0.25 \%$, BEJN $45.1 \pm$ $0.8 \%, \mathrm{JN} 60.9 \pm 7.6 \%$ ) (see the supplementary materials at http://www.rcjournal.com). High mask leak significantly lowered aerosol delivery compared to low mask leak $(P<$ .001). With the nebulizers at the ventilator, the VMN delivered $7.3 \pm 1.8 \%$ at high mask leak, whereas the BEJN delivered $2.3 \pm 0.4 \%$ and the $\mathrm{JN}$ delivered $2.2 \pm 1.1 \%$. Notably, there was no significant difference in aerosol delivery between the 2 nebulizer positions (At low leak, $P=.13$ and at high leak $P=.38$ ). Nebulizers placed at the ventilator outlet delivered equal or more aerosol than when the nebulizers were placed in the pre-mask position. At low mask leak, the VMN delivered $22.8 \%$ at the ventilator outlet versus $23.7 \%$ at the pre-mask position, whereas the JN delivered $8.1 \%$ at the ventilator outlet versus $4.2 \%$ at the premask position. Aerosol delivery with nebulizers in the premask position was similarly decreased at high mask leak, with the VMN delivering $23.7 \pm 5.1 \%$ at low mask leak versus $6.8 \%$ at high mask leak and the $\mathrm{JN}$ delivering $4.2 \pm$ $1.7 \%$ at low mask leak versus $1.5 \pm 0.7 \%$ at high mask leak.

\section{Aerosol Delivery and Losses Throughout the NIV Circuit}

The delivery of aerosol throughout the circuit with the nebulizer at the ventilator outlet and at the pre-mask position is presented in Figure 3. Values of activity as a percentage of nebulizer charge were shown in sequence while moving from the nebulizer to the inhaled mass filter. Only one of the following filters was in use at any given time, depending upon the test that was being conducted. When the nebulizer was placed at the ventilator outlet, aerosol first reached the pre-leak filter (before the leak port), then the pre-mask filter (between the mask and the leak port), followed by some aerosol deposition on the mask and face, and finally the remaining particles were deposited on the inhaled mass filter (inside the head). With the nebulizer in the pre-mask position, aerosol first encountered the pre-mask filter, then the mask and face, and finally the inhaled mass filter. Between the preleak and pre-mask filters, some aerosol was released through the leak port. From the pre-mask position to the inhaled mass filter, aerosol was either deposited onto the face and mask or lost through the face mask leak. Aerosol losses throughout the NIV circuit were indicated by the gradual decline in aerosol delivery from one filter position in the circuit to the next. Aerosol deposition in the circuit and nebulizer adapters was also measured (see the supplementary materials at http://www.rcjournal.com). 

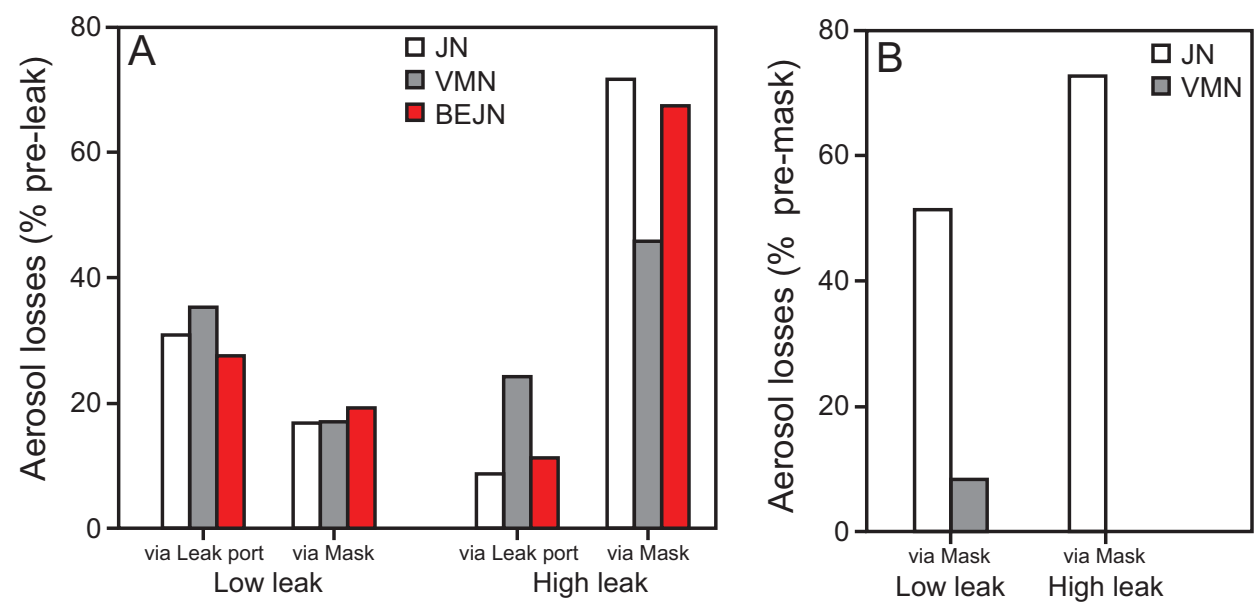

Fig. 4. Aerosol loss expressed as a percentage of the average aerosol deposited onto (A) the pre-leak filter or (B) the pre-mask filter. Because these numbers reflect an average, no standard deviation bars were plotted. Aerosol loss through the leak port and mask leak are shown in ( $A$ ) with the nebulizer at the ventilator outlet. Because the nebulizer placed in the pre-mask position bypasses the leak port, only losses via the mask leak are reported in $(B)$. The maximum amount of aerosol available through the circuit is the amount of aerosol deposited onto $(A)$ the preleak filter or $(\mathrm{B})$ the pre-mask filter. Beyond the pre-leak filter, aerosol was vented through either the leak port or the mask leak and the effect of a low mask leak (15-20 L/min) versus a high mask leak (55-60 L/min) is presented. With the nebulizer in the ventilator outlet, 28-36\% of aerosol was lost through the leak port and $15-20 \%$ was vented through the mask leak at low leak. At high mask leak, less aerosol was lost through the leak port, but significantly more was vented through the mask leak (46-72\%). When the nebulizer was placed in the pre-mask position, the JN lost $51.9 \%$ at low leak versus $73.3 \%$ at high leak, whereas the VMN lost $8.4 \%$ at low leak versus $0 \%$ through the mask leak. Aerosol losses due to leaks may be approximated from these relationships: (1) leak port loss = pre-leak filter deposition - pre-mask filter deposition; (2) mask leak loss $=$ pre-mask filter deposition - (inhaled mass filter deposition + face and mask deposition). Normalizing the results based on the percentage of aerosol deposited onto $(A)$ the pre-leak filter and $(B)$ the pre-mask filter accounted for the aerosol circuit deposition. $\mathrm{VMN}=$ vibrating mesh nebulizer; $\mathrm{JN}=$ jet nebulizer; $\mathrm{BEJN}=$ breath-enhanced jet nebulizer.

\section{Aerosol Loss Locations}

Loss of aerosol through the leak port, defined as the difference between the average pre-leak and pre-mask filter deposition, is shown in Figure 4. Similarly, the amount of aerosol lost through the mask leak is represented as the difference between the pre-mask filter and the sum of the aerosol deposited on the inhaled mass filter and the aerosol deposited on the face and mask. Figure 4 shows the aerosol losses from the leak port and mask leak, both at a low mask leak and at a high mask leak for both nebulizer positions. Results are represented as either percent pre-leak (when nebulizer was placed at the ventilator outlet) or pre-mask (when the nebulizer was placed in the pre-mask position). The purpose was to normalize for differences in nebulizer efficiency (ie, aerosol deposition onto the circuit and nebulizer adapters) and to provide a comparison between the losses between the 2 locations. With the nebulizers at the ventilator outlet, there was higher circuit deposition with the VMN (5-20\% of nebulizer charge) compared to the JN (2-8\%) and BEJN (2-6\%) (see the supplementary materials at http://www.rcjournal.com). With nebulizer at the ventilator outlet, statistical analysis indicated that a significant amount of aerosol was lost through the leak port $(P=.031)$ and through the mask leak $(P=.031)$. At low mask leak, the leak port had an average aerosol loss of $28-36 \%$ versus 9-24\% at high leak, whereas the amount of aerosol lost through the mask leak was 15-20\% at low leak versus 46$72 \%$ at high leak. When the nebulizer was placed in the pre-mask position, only the mask leak was quantified because the leak port was bypassed. At low leak, the JN lost an average of $51.9 \%$ while the VMN lost $8.4 \%$ through the mask leak. At high leak, the JN lost an average of $73.3 \%$, whereas the VMN had no recorded losses. Although the VMN had no or minimal losses via the face mask leak, most of the aerosol was deposited onto the face and mask, as described in the next section.

\section{Face and Mask Deposition}

The amount of aerosol deposited onto both the face and mask combined is shown in Figure 5. Results were plotted as either a percentage of averaged pre-leak filter activity (when the nebulizer was placed at the ventilator outlet) or as a percentage of averaged pre-mask filter activity (when the nebulizer was placed in the pre-mask position) to assess the deposition of available aerosol at each point in the circuit. With the nebulizers at the ventilator outlet, $10.6-26.2 \%$ at low leak versus $8.7-20.3 \%$ at high leak was deposited onto the face and mask, respectively. Comparatively, with nebulizers at the pre-mask position, the $\mathrm{JN}$ deposited $21.9 \%$ at low mask leak versus $22.2 \%$ at high mask leak, whereas the VMN deposited $42.2 \%$ at low mask leak versus $88.2 \%$ at 


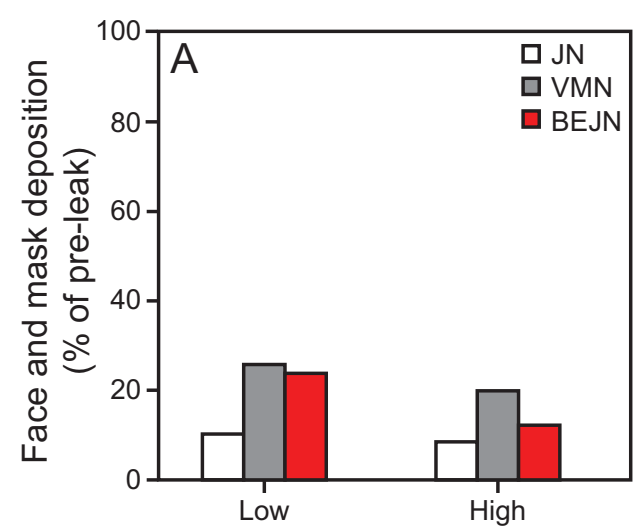

Amount of mask leak

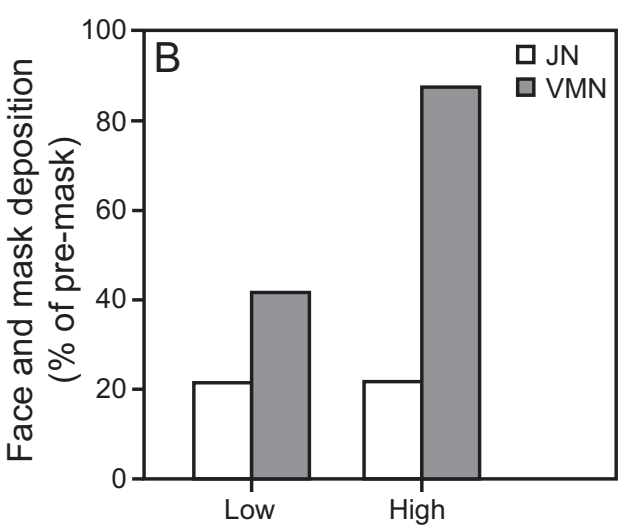

Amount of mask leak

Fig. 5. Face and mask deposition with the nebulizer at $(A)$ the ventilator outlet versus (B) pre-mask. The amount of aerosol deposited onto the face and mask for each experiment is expressed as either a percentage of the average aerosol deposited on the pre-leak filter (when the nebulizer was placed at the ventilator outlet) or as a percentage of average aerosol deposited on the pre-mask filter (when the nebulizer was placed in the pre-mask position). Because the numbers reflect an average, no standard deviation bars were plotted. More aerosol was deposited onto the face and mask when the nebulizer was placed in the pre-mask position compared to the ventilator outlet. With the nebulizer at the ventilator outlet, face and mask deposition was 10-26\% across the nebulizers. In contrast, approximately $42 \%$ of aerosol that reached the pre-mask filter from the VMN was deposited onto the face and mask with low leak versus $88 \%$ at high leak when the nebulizer was placed in the pre-mask position. VMN = vibrating mesh nebulizer; $\mathrm{JN}=$ jet nebulizer; $\mathrm{BEJN}=$ breath-enhanced jet nebulizer.
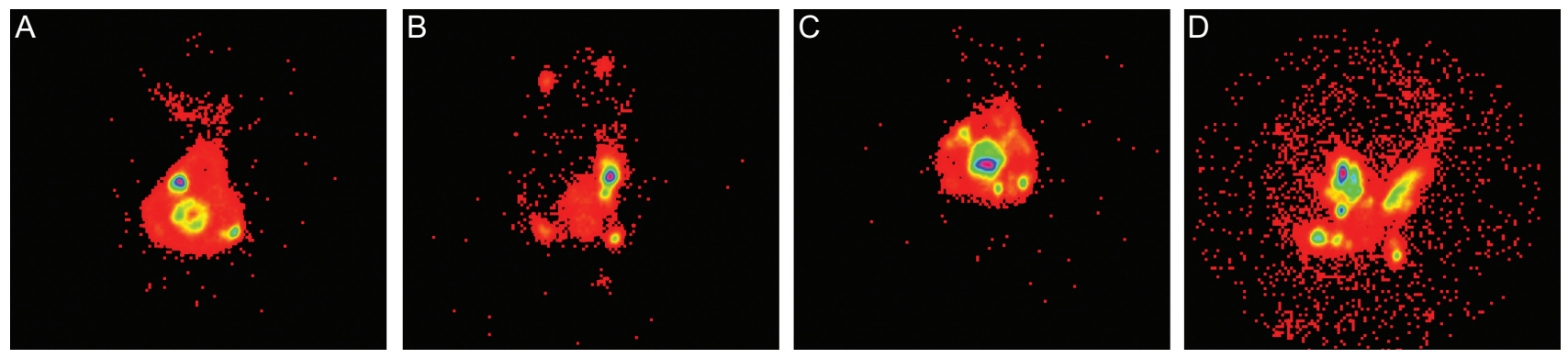

Fig. 6. Face and mask deposition of aerosol with the VMN, as percent nebulizer charge, with low mask leak. When the VMN was placed at the ventilator outlet (A and B), 11.3\% of nebulizer charge was deposited onto the mask alone (A) vs $6.1 \%$ of nebulizer charge deposited onto the face (B). In contrast, with the VMN placed in the pre-mask position ( $C$ and $D), 15 \%$ of the nebulizer charge was deposited onto the mask alone (C) vs $12.2 \%$ of nebulizer charge onto the face (D). VMN = vibrating mesh nebulizer.

high mask leak. As a percentage of nebulizer charge, VMN deposited 50\% onto the face and mask at high mask leak.

Figure 6 illustrates gamma camera images for an additional experiment to quantify deposition on the mask and the mannequin face separately. The VMN was used for this experiment because it had the highest aerosol output. At low mask leak, the NIV mask alone received $11.3 \%$ of the nebulizer charge when the nebulizer was placed in the ventilator outlet position, while a slightly higher amount (15\%) was deposited on the mask when the nebulizer was placed in the pre-mask position. Results were different for face deposition. For the face alone, with low mask leak, twice the amount was deposited onto the face when the nebulizer was placed in the pre-mask position (12.2\%) compared to the ventilator outlet position $(6.1 \%)$. With the nebulizer in the pre-mask position, the amount of aerosol deposition onto the face further increased to $50 \%$ with high mask leak (55-60 L/min).

\section{Aerogen VMN Behavior}

Multiple experiments with the Aerogen VMN had incomplete nebulization, defined by premature cessation of nebulization indicated by a residual radioactivity of $>10 \% .{ }^{12}$ Eighteen of $69(26 \%)$ VMN experiments failed to complete nebulization and were repeated. There were no failed experiments with either the BEJN or the JN. At the beginning of experiments, we started with 4 new VMNs. However, early on in our experiments, we noted that 1 of the 4 VMNs ceased aerosol output completely, with a $100 \%$ nebulizer residual (VMN 4 in Fig. 7). As a result, another VMN was substituted into the protocol. Furthermore, 


\section{Face Mask Leak and Aerosol Delivery in NIV}

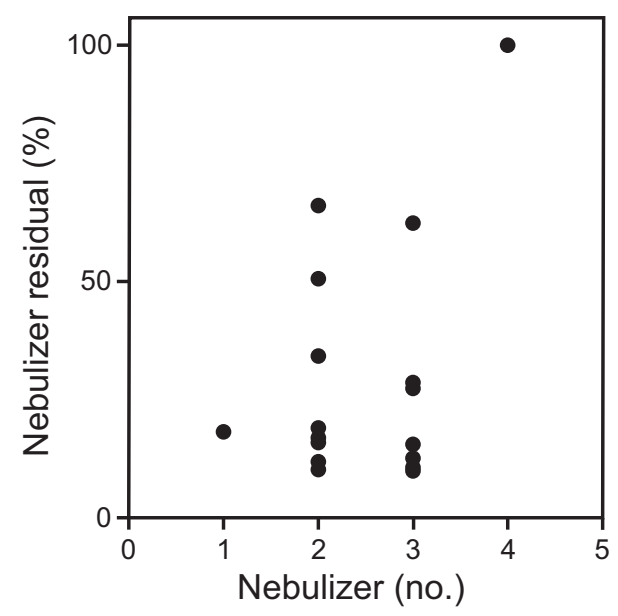

Fig. 7. Scatter plot of incomplete vibrating mesh nebulizer experiments in which nebulizer residual was $>10 \%$. Nebulizer residual as a percentage of initial nebulizer charge is plotted on the $y$ axis against individual vibrating mesh nebulizer units, numbered 1-4 on the $x$ axis. Nebulizers 2 and 3 had the most random failures. Nebulizer 4 , although brand new, failed to nebulize (ie, had a $100 \%$ residual).

additional VMNs failed to complete nebulization 5 times in the pre-mask position during the high face mask leak condition. While the VMN was the most efficient of the 3 tested nebulizers, there was more inter- and intra-device variability between VMN units than among the BEJN or JN devices. Only the experimental results with completed nebulization (residual radioactivity $<10 \%$ ) were plotted in Figures 1-3. Additional factors contributing to variability are reflected by the wider SD error bars displayed for the VMN data in contrast to the other devices.

\section{Summary of Results}

Efficiency of aerosol delivery was affected by the type of nebulizer technology; VMN had the highest efficiency, followed by BEJN and the JN $(P=.001)$ (Fig. 2). High mask leak $(55-60 \mathrm{~L} / \mathrm{min})$ significantly decreased aerosol delivery $(P<.001)$ (Fig. 2). There was no difference in aerosol delivery between nebulizer placement at the ventilator outlet position versus the pre-mask position at low mask leak $(P=.13)$ or high mask leak $(P=.38)$ (Fig. 2, Fig. 3). Significant aerosol losses were seen through both the leak port and mask leak $(P=.031)$; however, efficiency of aerosol delivery to the inhaled mass filter was ultimately a function of mask leak (Fig. 4). The pre-mask nebulizer position resulted in higher aerosol deposition onto the face and mask (Fig. 5, Fig. 6).

\section{Discussion}

This study is the first in vitro assessment of aerosol delivery during NIV to incorporate a deliberate mask leak, both at low $(15-20 \mathrm{~L} / \mathrm{min})$ and high $(55-60 \mathrm{~L} / \mathrm{min})$ test conditions. In the presence of a face mask leak, there were generally no differences in aerosol delivery whether the nebulizers were at the pre-mask position (between leak port and mask) or at the ventilator outlet position. These results are in contrast to the overall consensus of the current literature, which suggests placement of the nebulizer between the leak port and the mask; however, testing in the literature was performed without a mask leak. ${ }^{1-6,13,14}$ Prior studies focused on the singular effects of the leak port instead of its combined effects with a mask leak. The incorporation of a mask leak in NIV changes the dynamics of aerosol delivery: higher mask leak causes increased flow in the circuit and decreased aerosol losses via the leak port. However, more aerosol is lost via the face mask leak, and nebulizer location affects how much aerosol may deposit on the face.

Placement of a nebulizer in the pre-mask position is challenging in a clinical setting because the nebulizer would need to be supported by a device or a caregiver to remain in its optimal configuration for the duration of treatment. In addition, in the pre-mask position, aerosol delivery may be affected by patient positioning or movement. The face deposition studies with the VMN at a low mask leak indicate that twice the amount of aerosol (12\% of nebulizer charge) is deposited on the face when the nebulizer is placed in the pre-mask position compared to the ventilator outlet position (6.1\% of nebulizer charge). On the basis of these results, nebulizers should be placed at the ventilator outlet, which is a more practical location than the pre-mask position with equal patient delivery.

The mass balance determination characterizes the delivery of aerosol throughout the NIV circuit and, ultimately, to the patient. Analysis of aerosol delivery to each filter position (ie, the pre-leak filter [prior to the leak port], the premask filter [between the leak port and the mask], and inhaled mass filter [inside the head]) defined the overall potential of each nebulizer, the amount of aerosol lost through the leak port, and the amount of aerosol lost through the mask leak. Our results indicate that the VMN was the most efficient of the 3 tested nebulizers, followed by the BEJN and the JN. However, there were significant aerosol losses through both the leak port and the mask leak. As the mask leak was increased from low $(15-20 \mathrm{~L} / \mathrm{min})$ to high $(55-60 \mathrm{~L} / \mathrm{min})$, the aerosol losses were reduced through the leak port with a substantially higher amount of aerosol lost through the mask leak (Fig. 4). These results indicate that while a higher mask leak was able to move particles past the leak port to the pre-mask filter, more aerosol was ultimately lost through the mask leak.

These findings suggest that clinicians should focus on minimizing mask leak when treating patients with aerosols on a NIV device. In our model, placing the mask on the mannequin consistently produced a leak of $15-20 \mathrm{~L} / \mathrm{min}$ as shown on the NIV device's monitor. If the patient moves or the leak is greater, aerosol delivery will likely be minimal. The clinical effects of mask leak on noninvasive ventilators 


\section{Face Mask Leak and Aerosol Delivery in NIV}

have, thus far, been studied only on CPAP devices and automatic positive airway pressure devices for sleep apnea patients. Montesi et al ${ }^{15}$ reported that air leak was associated with the development of acute central sleep apnea, while Coller et al ${ }^{16}$ noted that increasing air leak in different automatic positive airway pressure devices prevented them from responding to respiratory events related to sleep apnea. Both studies highlight the importance of mask leak.

While the VMN proved to be the most efficient of the 3 studied nebulizers, $26 \%$ of the VMN studies had to be repeated due to incomplete nebulization, defined as a nebulizer residual of $10 \%$ or more. ${ }^{12}$ The rate of incomplete nebulization was similar to that reported by Gowda et al. ${ }^{12}$ Figure 7 graphically depicts the VMN experiments with nebulizer residual $>10 \%$ and the respective VMN unit. One VMN unit failed to nebulize completely, with a $100 \%$ nebulizer residual despite multiple attempts, and that unit was eventually discarded. As a result, more VMN devices were required to complete this study as compared to the BEJNs and JNs. Incomplete nebulization appeared to be random. In addition, the VMN exhibited considerable interand intra-device variability as shown by the larger standard deviation compared to the BEJN and JN (Fig. 2, Fig. 3).

\section{Limitations}

The Philips Respironics V60 is a single-limb, constantflow, dedicated noninvasive ventilator, and results from this study apply only to that ventilator and should not be extrapolated to other devices, such as conventional critical care ventilators when set up for aerosol delivery during NIV via a face mask. Similarly, these experiments were conducted on a system with the intentional leak port located in the delivery tubing rather than the mask. Systems with the intentional leak port in the mask may have different results. Other untested factors, such as humidification, may influence aerosol delivery. In conventional invasive mechanical ventilation, the addition of humidity has been shown to reduce aerosol delivery by approximately $50-75 \%{ }^{17}$

\section{Conclusions}

During NIV, face mask leak substantially reduces aerosol delivery. Placement of nebulizers at the ventilator outlet is as effective as placement at the pre-mask position. The ventilator outlet location is more convenient for both the patient and health care workers and decreases potential facial deposition.

\section{REFERENCES}

1. Michotte J-B, Jossen E, Roeseler J, Liistro G, Reychler G. In vitro comparison of five nebulizers during noninvasive ventilation: analysis of inhaled and lost doses. J Aerosol Med Pulm Drug Del 2014;27 (6):430-440.

2. Abdelrahim ME, Plant P, Chrystyn H. In-vitro characterisation of the nebulised dose during non-invasive ventilation. J Pharm Pharmacol 2010;62(8):966-972.

3. Peng Y, Dai B, Hu CX, Su J, Tan W, Zhao HW, et al. Which nebulizer position should be avoided? An extended study of aerosol delivery and ventilator performance during noninvasive positive pressure ventilation. Respiration 2018;95(3):145-153.

4. Calvert LD, Jackson JM, White JA, Barry PW, Kinnear WJ, O'Callaghan C. Enhanced delivery of nebulised salbutamol during non-invasive ventilation. J Pharm Pharmacol 2006;58(11):1553-1557.

5. Sutherasan Y, Ball L, Raimondo P, Caratto V, Sanguineti E, Costantino F, et al. Effects of ventilator settings, nebulizer and exhalation port position on albuterol delivery during non-invasive ventilation: an in-vitro study. BMC Pulm Med 2017;17(1):9.

6. Chatmongkolchart S, Schettino GPP, Dillman C, Kacmarek RM, Hess DR. In vitro evaluation of aerosol bronchodilator delivery during noninvasive positive pressure ventilation: effect of ventilator settings and nebulizer position. Crit Care Med 2002;30(11):2515-2519.

7. Branconnier MP, Hess DR. Albuterol delivery during noninvasive ventilation. Respir Care 2005;50(12):1649-1653.

8. White CC, Crotwell DN, Shen S, Salyer J, Yung D, Zheng J, et al. Bronchodilator delivery during simulated pediatric noninvasive ventilation. Respir Care 2013;58(9):1459-1466.

9. Aerogen Solo System Instruction Manual (P/N AG-AS3050-US, P/N 30-674 Rev H.). Dangan, Ireland: Aerogen, Ltd, 2017.

10. Sangwan S, Gurses BK, Smaldone GC. Facemasks and facial deposition of aerosols. Pediatr Pulmonol 2004;37(5):447-452.

11. Smaldone GC, Sangwan S, Shah A. Facemask design, facial deposition, and delivered dose of nebulized aerosols. J Aerosol Med 2007;20 (Suppl 1):S66-S77.

12. Gowda AA, Cuccia AD, Smaldone GC. Reliability of vibrating mesh technology. Respir Care 2017;62(1):65-69.

13. Hess DR. Aerosol therapy during noninvasive ventilation or high-flow nasal cannula. Respir Care 2015;60(6):880-893.

14. Dhand R. Aerosol therapy in patients receiving noninvasive positive pressure ventilation. J Aerosol Med Pulm Drug Deliv 2012;25(2):63-78.

15. Montesi SB, Bakker JP, Macdonald M, Hueser L, Pittman S, White DP, et al. Air leak during CPAP titration as a risk factor for central apnea. J Clin Sleep Med 2013;9(11):1187-1191.

16. Coller D, Stanley D, Parthasarathy S. Effect of air leak on the performance of auto-PAP devices: a bench study. Sleep Breath 2005;9(4):167175 .

17. Miller DD, Amin MM, Palmer LB, Shah AR, Smaldone GC. Aerosol delivery and modern mechanical ventilation. Am J Respir Crit Care Med 2003;168(10):1205-1209. 